Based on earlier studies of UDG-crystal structures in the absence of $\mathrm{DNA}^{7}$, Slupphaug et al. ${ }^{4}$ produced a mutant form of UDG that had a reduced catalytic efficiency and an increased affinity for DNA. The crystal structure of this enzyme bound to a 10-base-pair DNA duplex with a central U-G mismatch reveals that the UDG active-site groove interacts, for four consecutive nucleotides, with the DNA backbone of the strand in which the uracil is found. The backbone flanking the uracil is compressed, and the uracil, deoxyribose and $5^{\prime}$ phosphate are flipped out of the helix into the major groove. Here they are stabilized by interactions within an enzyme pocket that defines specificity for the removal of uracil but not of the four normal bases. The flipped uracil is positioned in the active site and the N-C 1 '-glycosylic bond is not observed - that is, this is an enzyme-product complex. In place of the nucleotide that has been displaced from the DNA helix is the side chain of the UDG residue 272 (normally a leucine, but an arginine in the mutant used here), which is inserted through the minor groove.

It has been proposed that base flipping to promote catalysis is common among a number of different DNA repair and base-modification enzymes ${ }^{2,3}$. But these enzymes each present interesting variations on the flipping theme. Escherichia coli photolyase is thought to flip out a cyclobutane pyrimidine dimer to allow the direct photoreversal of the dimer ${ }^{8}$. In contrast, when $\mathrm{T} 4$ endonuclease $\mathrm{V}$ removes dimers, it has been suggested that it doesn't flip the dimer itself, but rather the opposing undamaged 5 ' base. The flipped base produces a cavity in the DNA duplex that allows access to the dimer for the enzyme's catalytic residues'. It has also been proposed that T7 DNA ligase flips the AMP residue that it covalently links to the $5^{\prime}$ phosphate of nicked duplex DNA. In this case, the flipping occurs to prevent interference of AMP with subsequent ligation ${ }^{10}$. And the structures of two cytosine methyltransferases bound to DNA also reveal flipped bases ${ }^{11}$ that are methylated and then returned to the helix.

Structural data indicate that the processes by which these flipping enzymes recognize their substrate DNAs may also be different. Damaged DNA that is bound to $\mathrm{T} 4$ endonuclease $\mathrm{V}$ is sharply kinked at the central thymine dimer, and there is considerable deformation of the phosphate backbone ${ }^{9}$. Along with a dimer-induced conformational perturbation in the duplex DNA prior to binding, these features may define initial recognition. In the case of the sequence-specific methyltransferases ${ }^{11}$, the base flips into the minor groove and the vacated space is occupied by resi-

\section{Four pins for four petals}

DNA-binding proteins come in many shapes and sizes, reflecting the diversity of the DNA structures to which they attach. The crystal structure of one such protein Escherichia coli RuvA - has now been solved by John Rafferty and co-workers (J. B. Rafferty et al. Science 274, 415-421; 1996), and they have proposed a model for the binding of RuvA to its substrate, the Holliday junction.

The four-stranded Holliday junction is a central intermediate in genetic recombination, and movement of the junction point allows the generation of heteroduplex DNA in a reaction that is driven by the RuvB DNA helicase. The functions of RuvA are to target RuvB to the Holliday junction and to hold the junction in a square planar configuration. And now the crystal structure reveals just how this is done.

RuvA protein is known to assemble into tetramers, and Rafferty et al. show that the monomers are related by fourfold rotational symmetry that is reminiscent of a four-petalled flower. Protruding from the concave face of each monomer is a small, negatively

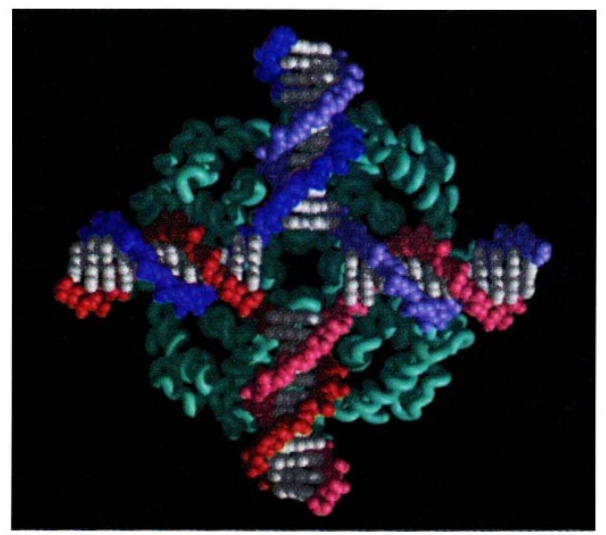

charged pin, and when a model of the tetrameric structure (green) is superimposed onto a model of the Holliday junction (DNA backbones shown in purple and red), the four pins fit neatly through the hole at the centre of the Holliday junction. These pins encourage the transient separation of the DNA strands within each arm of the junction because of repulsive interactions between the pins and the phosphate backbones. In this way, RuvA holds the DNA in the requisite conformation for processing by the RuvB helicase. dues that enter from the major groove.

Interestingly, just the opposite is true for UDG, which is not sequence specific. Residue 272 enters from the minor groove, and the flipped uracil occupies the UDG specificity pocket in the major groove $^{4}$. Does this suggest that UDG, and possibly other repair glycosylases, initially recognize their substrates by scanning the minor groove for subtle differences from normal geometry? This proposal has already been made for another class of non-sequence-specific DNA-processing enzymes, the DNA polymerases. For example, it has been suggested that DNA polymerase- $\beta$, the polymerase used for base-excision repair, may probe the positions of hydrogen-bond donor and acceptor groups in the DNA minor groove to discriminate between correct and incorrect base pairs ${ }^{12}$

The extremely low catalytic rate of Hha I methyltransferase is consistent with the possibility that methyltransferases simply capture cytosines that spontaneously unstack from their neighbours and flip into the minor groove of the helix ${ }^{3,11}$. Based on the much higher catalytic rate of UDG and the new structure of the UDG-DNA complex ${ }^{4}$, Slupphaug et al. suggest that UDG actually facilitates base flipping into the major groove. It is thought that UDG uses certain amino acids to compress the DNA backbone and 'push' the uracil base out of the helix, while other amino acids 'pull' or stabilize uracil in the flipped-out configuration. This leads to an obvious question: does UDG flip out every base that it encounters and then capture only those that can be accommodated by the specificity pocket, or does it flip out only the base that it is designed to repair? The rich detail of the new UDG-DNA co-crystal structure provides exciting possibilities for experiments to address this and other questions on the mechanism of action of this exquisitely specific and critical DNArepair enzyme.

Thomas A. Kunkel and Samuel H. Wilson are in the Laboratory of Structural Biology, National Institute of Environmental Health Sciences, PO Box 12233, Research Triangle Park, North Carolina 27709, USA.

\footnotetext{
. Bennett, S. E., Sanderson, R. J. \& Mosbaugh, D. W. Biochemistry 34, 6109-6119 (1995).

. Roberts, R. J. Cell 82, 9-13 (1995).

3. Pearl, L. H. \& Sawa, R. Trends Biochem. Sci. 20 421-426 (1995)

Slupphaug, G. et al. Nature 384, 87-92 (1996).

. Lindahl, T. Nature 362, 709-715 (1993).

6. Seebers, E Eide L \& Bjoras, M Trends Biochem Sci. 20, 391-397 (1995).

Moi, C. D. et al. Cell 80, 869-878 (1995)

8. Park, H. W., Kim, S.-T., Sancar, A. \& Deisenhofer, J. Science 268, 1866-1872 (1995).

Vassylyev, D. G. et al. Cell 83, 773-782 (1995).

10. Subramanya, H. S., Doherty, A. J., Ashford, S. R. \& Wigley, D. B. Cell 85, 607-615 (1996).

11. Nelson, H. C. M. \& Bestor, T. H. Chem. Biol. 3, 419-423 (1996).

12. Beard, W. A. et at. J. Biol. Chem. 271, 12141-12144
} (1996). 